\title{
Positioning Study of the Robot Vision based on Geometry
}

\author{
Jun Sun \\ College of Civil Engineering and Architecture, Guizhou University of Engineering Science, Bijie, Guizhou, 551700, China \\ Junsun791205@gmail.com
}

\begin{abstract}
The robot's eyes through the background difference method were used to find broke into the visual range of a moving object and track and monitor the moving object. On the basis of geometry and according to the distance and deflection angle of the robot eyes positioning, the objects were captured and tracked by robots eyes. Geometry method precision was low, but simple calculation processing was quick. Thus, it can effectively meet the robot eyes preliminary positioning of the fast moving target.
\end{abstract}

Keywords-moving object; tracking capture; geometric positioning.

\section{INTRODUCTION}

The spatial position of an object can be estimated with feelings or experience, when it is being observed with human eyes. Robot's eyes can have the same function and also will much more accurately estimate the spatial position of objects than human eyes. Fruitful domestic and foreign results have been achieved in the study of robot binocular positioning. In this paper, some new breakthroughs were expected to make in the study of robot binocular positioning on the basis of summarizing the predecessors' studies.

In April 2010, Hongguang SHI et al [1] deeply studied the camera calibration and stereo matching in the key binocular stereovision technology and established an experimental platform for binocular vision system using the improved plane calibration method and the stereo matching algorithm based on the polar constraint and gray correlation, in order to program target positioning and implement the positioning on the target object. In May 2010, Zixiao ZHOU et al [2] configured intrinsic parameters into the software system of simulated robot platform by experimentally solving both intrinsic and extrinsic parameters of camera lens and using software simulation algorithm and the correctness of the calibration results, and also implement the binocular stereovision positioning function in the system. In April 2011, Qian SHANG et al [3] proposed a Pan-Tilt corner based extrinsic parameter estimation method which could accurately complete extrinsic parameter estimation in case of camera rotation and enhance the visual function of the robot, and also implemented target recognition and positioning based on the improved binocular vision system using Grandar robot system, and then used the result as a basis for controlling the corresponding movements of robot, so that the capture of the target objects was finally implemented. In May 2011, Yuan-jie CHEN et al [4] designed a camera seal structure according to underwater liquid environment and introduced high-order distortion parameters to the calibration of camera for obtaining the intrinsic and extrinsic parameters under the same underwater environment, and then proposed a forward collimation based simple localization algorithm according to the target point solving method for the sight intersection in the traditional complex binocular camera. In June 2012, Fuling ZHANG et al [5] determined the coordinates of the targets' geometric center by establishing a graph coordinate transformation model and using the least square method, and then provided the targets according to the geometric relationship and confirmed the mathematical model and calculation method for the coordinate transformation of the two fixed cameras' relative positions. In March 2013, Huigui QIU et al [6] calculated the 3d coordinates marked on the positioned target using the matching results and the least square method. In June 2013, Yankai CHAO et al [7] calibrated the binocular camera using the plane template method and constructed a robot coordinate system, thus finishing the positioning on the target object. Binocular camera after calibration can acquire the position of target object in space coordinate system; the coordinate value of target object in the robot world coordinate system is defined through coordinate transformation, which is the important data for robot to implement target object servo tracking and capturing. In September 2013, Min XU et al [8] proposed a binocular stereovision based method to track and position the moving objects in real time, which effectively integrated stereo matching algorithm with target tracking algorithm; they calculated the 3d coordinates of the target using binocular stereovision technology when the target was being tracked in real time, so as to realize accurate positioning.

The traditional robot binocular positioning algorithm possessed a high accuracy, but it reduced the ability of robot to rapidly capture the moving targets due to the complex calculation process. In this paper, the binocular positioning on the target object was implemented by background difference and geometric calculation on the basis of reference [15]. The response velocity of the robot vision was effectively improved, because the calculation method was simple and fast.

\section{THE BINOCULAR GEOMETRIC POSITIONING PRINCIPLE OF ROBOT}

The two eyes of robot are actually two independently operated cameras, which discover and track the objects into their visual scope using background differencing [15]. As shown in Fig.1, points A and B were the positions of the 
robot's two eyes (i.e. two cameras); point $O$ was the center of line segment $|A B|$; point $C$ was the position of the object. Robot automatically established a 3d coordinate system with $O$ as the origin when it locked the object, as shown in Fig.1.

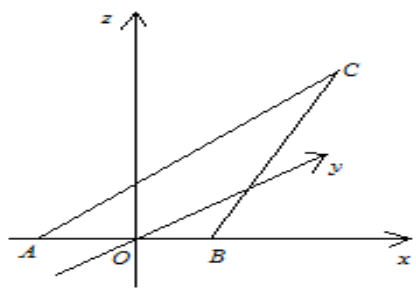

Figure 1 . The eyes of the robot and the position of the object

Robot binocular positioning was to the $3 \mathrm{~d}$ coordinates of point $C$ in the coordinate system as shown in Fig.1.

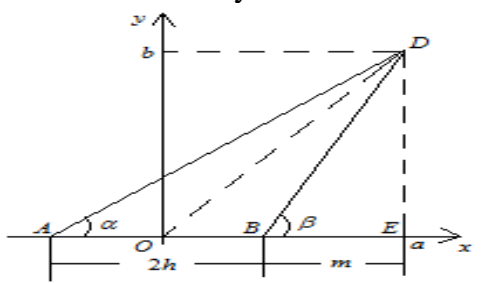

Figure 2. The projection of the object in plane xoy

In Fig.2, points $A$ and $B$ were where the eyes of the robot are placed; point $D$ was the projection point of object $C$ on plane xoy; point $E$ was the projection point of point $D$ on $\mathrm{x}$-axis. $\alpha$ was the intersection angle of the camera at point $A$ on plane xoy; $\beta$ was the intersection angle of the camera at point $B$ on plane xoy. The length of line segment $|\mathrm{AB}|$ was $2 h$; the length of line segment $|\mathrm{BE}|$ was $m$. The coordinates (a, b) of point $D$ were finally confirmed by the values of $\alpha, \beta \& h$.

$$
\begin{gathered}
\tan \alpha=\frac{|D E|}{|A E|}=\frac{b}{2 h+m} \\
\tan \beta=\frac{|D E|}{|B E|}=\frac{b}{m} \\
m=\frac{2 h \tan \alpha}{\tan \beta-\tan \alpha}
\end{gathered}
$$

Thus,

$$
\left\{\begin{array}{l}
a=\frac{2 h \tan \alpha}{\tan \beta-\tan \alpha}+h \\
b=\frac{2 h \tan \alpha}{\tan \beta-\tan \alpha} \tan \beta
\end{array}\right.
$$

Then, the coordinates $(\mathrm{a}, \mathrm{b})$ of object $C$ on plane xoy were obtained.

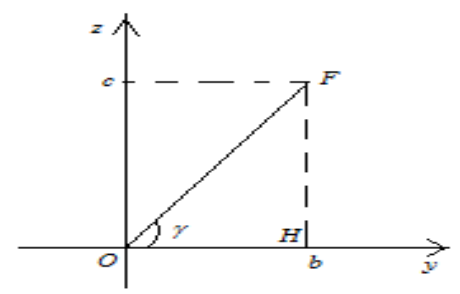

Figure 3. The projection of object $C$ on plane xoy

In Fig.3, point $F$ was the projection point of object $C$ on plane $x o y ; \gamma$ was the droop angle of the cameras at $A$ and $B$ respectively. The mean value of the droop angles of the two cameras was chosen for $\gamma$, because these angles were different to some extent.

$$
\tan \gamma=\frac{|F H|}{|O H|}=\frac{c}{b}
$$

The following equation was obtained by referring to equation (3).

$$
c=b \tan \gamma=\frac{2 h \tan \alpha}{\tan \beta-\tan \alpha} \tan \beta \tan \gamma
$$

Thus, the position (a, b, C) of object $C$ in the coordinate system was determined, as shown in Fig.1.

$$
\left\{\begin{array}{l}
a=\frac{2 h \tan \alpha}{\tan \beta-\tan \alpha}+h \\
b=\frac{2 h \tan \alpha}{\tan \beta-\tan \alpha} \tan \beta \\
c=\frac{2 h \tan \alpha}{\tan \beta-\tan \alpha} \tan \beta \tan \gamma
\end{array}\right.
$$

Where, $h$ was a known a fixed value; the values of $\alpha, \beta, \gamma$ were acquired by the measuring device inside the robot eyes.

\section{THE IMPLEMENTATION STEPS AND FLOW CHART}

Step 1: Two identical cameras were chosen and placed on a free rotating bracket and also used as the two eyes of the robot. Then, the distance $h$ between the two cameras was measured.

Step 2: Two cameras were in a parallel, static forward state and also turned on. They were allowed to take out two images to compare at a time interval $\Delta \mathrm{t}$.

Step 3: The object into the robot binocular vision was tracked and captured using the method in reference [15].

Step 4: The values of the two cameras' deflection angles $\alpha, \beta, \gamma$ were acquired in real time through the measuring instrument installed on the free rotating bracket.

Step 5: The measured values of $\alpha, \beta, \gamma$ and the value of $h$ were substituted into equation (6). Then, the location of the moving object was preliminarily positioned.

Step 6: The positioning value obtained in the above measurement was substituted into SVM or neural network. Then, the trend of the fast moving object was predicted. 
Finally, the moving object was quickly, accurately positioned using precision positioning device.

The flow of robot binocular to position a moving object using geometry method was as follows:

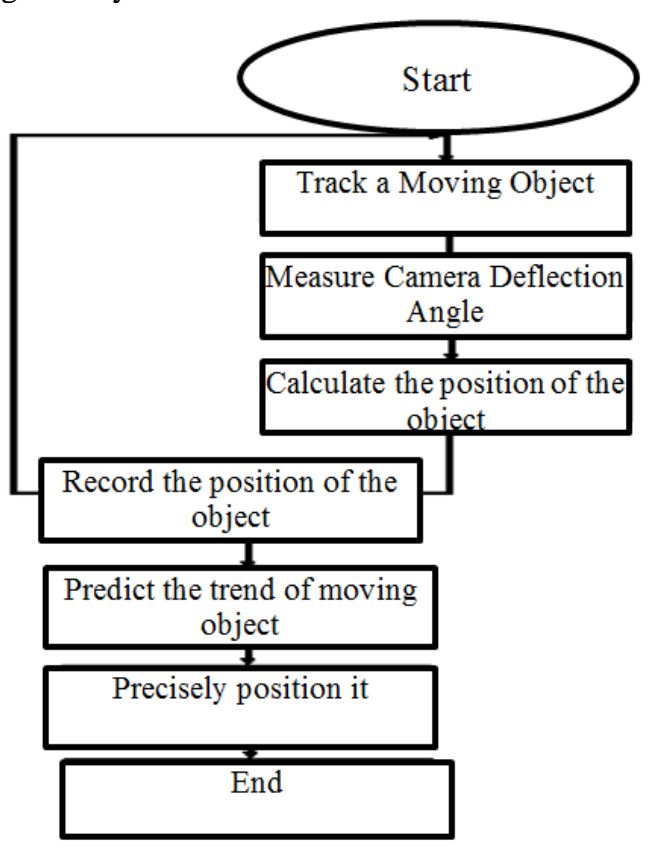

Figure 4. The binocular geometric positioning flow

\section{CONCLUSION}

The experimental result showed that the calculation process was simple and quick if robot tracked and positioned a moving object using geometry method, and also the speed and accuracy of robot binocular to position a fast moving object were effectively improved in the traditional algorithm. In the meantime, the application of geometry method to tracking and positioning a moving object is an effective backup positioning method once all the positioning systems built in robot fail to work. It makes robot still capable of maintaining its task execution if other positioning systems suddenly fail to work.

\section{ACKNOWLEDGEMENT}

Bijie University Joint Fund Project (Guizhou science joint J LKB[2012]08)

\section{REFERENCES}

[1] Hongguang SHI, Feng-sheng ZHANG, Chun-lan ZHENG. Design on Object Detection System based on Binocular Vision [J]. Modern Instruments, 2010.4(1): 45-47.

[2] Zixiao ZHOU, Xiaolin ZHAO, et al. Binocular Stereo Location on Emulator Robot [J]. Video Engineering, 2010.8(34):106-109.

[3] Qian SHANG, Qiu-qi RUAN, Xiaoli LI. Target Recognition and Location based on Binocular Stereo Vision [J]. CAAI Transactions on Intelligent Systems, .2011.4(6): 303-311.

[4] Yuanjie CHEN, Kangwu ZHU, et al. Binocular Vision Based Locating System for Underwater Inspection [J]. Mechanical \& Electrical Engineering Magazine, 2011.5(28): 567-573.

[5] Fuling ZHANG, Zhangkun CHENG, et al. Binocular Orientation Based On Coordinate Alternations [J]. Journal of Weinan Teachers College, 2012.6(27): 8-12.

[6] Huigui QIU, Xin CHEN. Collection and Analysis of 3D Coordinates Tag in Binocular Stereo Vision Positioning [J]. Journal of Minjiang University, 2013.2(34): 98-101.

[7] Yankai CHAO, Yu-lin XU, et al. Robots Binocular Calibration and Target Location Based on Planar Template [J]. Computer Technology and Development, 2013.6(23): 27-34.

[8] Min XU, Zhongwei ZHOU. Moving Object Tracking and Positioning Method based on Binocular Stereo Vision [J]. Manufacturing Automation, 2013.9(35): 69-72.

[9] Jun SUN. Study on the Remote Sensing Image Geometric Correction Algorithm based on Support Vector Machine (SVM) [D]. Xi'an: Xi'an University of Science and Technology, 2011.6.

[10] Jun SUN, Qi LI, Herui LI. Remote Sensing Image Approximate Geometric Correction Based On Matlab7.4 [J]. Computer Applications and Software, 2012.9 (29): 249-251.

[11] Kenneth R. Castleman. Digital Image Processing [1] [M]. Beijing: The Electronic Industrial Press, 2011.

[12] Min YAO, et al. Digital Image Processing [M]. Beijing, Mechanical Industry Publishing House, 2011.

[13] Weiguo LIU. Matlab Programming and Applications (ed.2) [M]. Beijing. Higher Education Press, 2006.

[14] Sunjun. Study on the Construction of 3-dimensional Image by Support Vector Machine [C], International Conference on Technology Management and Engineering Science. NGVEverlag. 2013.24.

[15] Sunjun. Study on Mobile Object Tracking and Intercept Technology Research[C], International Journal of Technology Management. International Science \& Technology Press, 2014.08. 